\title{
Diacronie
}

Studi di Storia Contemporanea

$\mathbf{N}^{\circ} 27,3$ | 2016

Stato, costituzione e democrazia

\section{Documenti inediti per la cronaca e per la lettura storica del Sessantotto napoletano}

\section{Carmine Marino}

\section{(C) OpenEdition}

\section{Journals}

\section{Edizione digitale}

URL: http://journals.openedition.org/diacronie/4260

DOI: 10.4000/diacronie.4260

ISSN: 2038-0925

\section{Editore}

Association culturelle Diacronie

\section{Notizia bibliografica digitale}

Carmine Marino, « Documenti inediti per la cronaca e per la lettura storica del Sessantotto

napoletano », Diacronie [Online], № 27, 3 | 2016, documento 4, Messo online il 29 septembre 2016 ,

consultato il 20 avril 2019. URL : http://journals.openedition.org/diacronie/4260 ; DOI : 10.4000/

diacronie.4260 


\title{
Diacronie
}

\section{Documenti inediti per la cronaca e per la lettura storica del Sessantotto napoletano}

\author{
Carmine MARINO *
}

Sulla scia dei moti di contestazione studentesca che investirono le principali città italiane nel biennio 1968-69, le maggiori organizzazioni universitarie napoletane (il Movimento Studentesco di Architettura e la Sinistra Universitaria) cercarono di proiettare le ragioni del proprio malcontento (l'inadeguatezza dell'assetto edilizio, incapace di soddisfare il crescente afflusso di studenti, l'autoritarismo del corpo docente) sullo sfondo dei problemi sociali che affliggevano la città di Napoli. Tuttavia, la mancanza di una strategia condivisa e la difficile interazione tra $i$ vertici del movimento ed i settori più moderati della comunità studentesca indebolirono il fronte della contestazione, sancendone pertanto il declino.

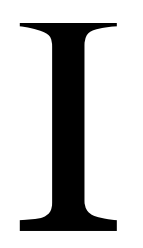

processi di mobilitazione studentesca che, nel biennio 1967-68, animarono i principali atenei italiani coinvolsero - seppure con modalità, tempi ed esiti differenti - anche le università del Mezzogiorno, a cominciare dalla Federico

II di Napoli. Gli studi dedicati al movimento partenopeo e le iniziative a scopo commemorativo promosse in tempi relativamente recenti hanno senz'altro contribuito a ricostruire una pagina altrimenti dimenticata della storia meridionale, proiettando i disagi di larghi settori della comunità accademica ed universitaria sullo sfondo di una città ancora alle prese con gli effetti delle spregiudicate politiche speculative perseguite dall'amministrazione Lauro. D'altra parte, queste ricerche e - più in generale - le pubblicazioni sul Sessantotto italiano ${ }^{1}$ non hanno offerto sufficienti chiavi di lettura che

\footnotetext{
${ }^{1}$ Non si contano gli studi, gli approfondimenti e le ricerche che hanno preso in esame gli anni della contestazione universitaria e le sue implicazioni. In questa sede, si segnalano alcuni dei contributi più interessanti: AGOSTI, Aldo, PASSERINI, Luisa, TRANFAGLIA, Nicola (a cura di), La cultura e i luoghi del '68, Milano, Franco Angeli, 1991; BALESTRINI, Nanni, MORONI,
} 
consentissero di inquadrare adeguatamente le vicende del movimento napoletano nella più ampia cornice delle agitazioni promosse dai centri universitari capofila (Trento, Milano, Torino), evidenziando altresì le ragioni che ne decretarono il declino e la conseguente estinzione. Ad ogni buon conto, è comunque possibile formulare una prima conclusione, per quanto inevitabilmente sommaria: se le centrali studentesche attive nelle grandi città del Nord Italia tentarono di uscire dal loro mondo per aprire un canale di dialogo con il mondo operaio in lotta, palesando oltretutto evidenti limiti sul piano strategico ed operativo, le avanguardie del movimento napoletano rimasero sostanzialmente intrappolate in uno sterile dibattito ideologico che precluse ogni possibilità di tradurre il pensiero - per la verità piuttosto disomogeneo e confuso - in azione.

Le prime mobilitazioni nelle università italiane - che risentirono senz'altro dell'influenza esercitata dai principi del cattolicesimo progressista, a cui si sommarono le epocali conquiste del Concilio Vaticano II (1962-1965) ${ }^{2}$ - si presentarono come il tentativo, non sempre omogeneo e coerente, di avviare un'opera di «democratizzazione dal basso» che mettesse in discussione la «stessa struttura di potere su cui si fondava l'istituzione universitaria, sulla base della constatazione che sulla gestione e l'impostazione sia della didattica che dei percorsi formativi gli studenti non avevano

Primo, L'orda d'oro. 1968-1977: la grande ondata rivoluzionaria e creativa, politica ed esistenziale, Milano, Feltrinelli, 1997; BRAVO, Anna, A colpi di cuore. Storie del Sessantotto, Roma-Bari, Laterza, 2008; FLORES, Marcello, DE BERNARDI, Alberto, Il Sessantotto, Bologna, Il Mulino, 2003; LUMLEY, Robert, Dal '68 agli anni di piombo. Studenti e operai nella crisi italiana, Firenze, Giunti, 1997; MARINO, Giuseppe Carlo, Biografia del Sessantotto. Utopie, conquiste, sbandamenti, Milano, Bompiani, 2004; PIZZORNO, Alessandro, REYNERI, Emilio, REGINI, Marino, REGALIA, Ida, Lotte operaie e sindacato: il ciclo 1968-1972 in Italia, Bologna, Il Mulino, 1978; VENTRONE, Angelo, "Vogliamo tutto". Perché due generazioni hanno creduto nella rivoluzione, Roma-Bari, Laterza, 2012. Per un bilancio complessivo sulle trasformazioni economiche e sociali che investirono l'Italia all'indomani dei fermenti sessantottini, è doveroso menzionare le seguenti monografie: CRAINZ, Guido, Autobiografia di una Repubblica. Le radici dell'Italia attuale, Milano, Feltrinelli, 2012; CRAVERI, Piero, Storia d'Italia. La Repubblica dal 1958 al 1992, Torino, Utet, 1995; DE LUNA, Giovanni, Le ragioni di un decennio. 1969-1979. Militanza, violenza, sconfitta, memoria, Milano, Feltrinelli, 2009; MALGERI Francesco, PAGGI, Leonardo, L'Italia repubblicana nella crisi degli anni Settanta. Partiti e organizzazioni di massa, Soveria Mannelli (CZ), Rubbettino, 2003. Tra le monografie che hanno esaminato le vicende del Sessantotto italiano in rapporto agli analoghi movimenti contestatari, è degno di interesse il lavoro di TOLOMELLI, Marica, Il Sessantotto. Una breve storia, Roma, Carocci, 2008.

2 «Le particolari circostanze in cui [il Concilio] si svolse, caratterizzate nei primi anni Sessanta da grandi aperture culturali e politiche, l'accelerata maturazione di cui i padri conciliari [...] furono protagonisti, la spinta inizialmente impressa da Giovanni XXIII, la sensazione diffusa di una grave crisi della Chiesa cui occorreva porre rimedio, determinarono il prevalere di un indirizzo di rinnovamento, perseguito da una maggioranza guidata da prelati francesi, tedeschi, belgi, olandesi, e in cui si manifestavano le esigenze presenti da alcuni decenni nel sottosuolo di una parte del Cattolicesimo europeo». VERUCCI, Guido, La Chiesa cattolica in Italia dall'Unità a oggi, Roma-Bari, Laterza, 1999, p. 81. 
alcun controllo»3. In breve tempo, però, le campagne di dissenso promosse nei singoli atenei mirarono ad un disegno decisamente più ambizioso, ovvero «passare da una fase di tipo sindacale, cioè di difesa di interessi essenzialmente corporativi, a una fase esplicitamente politica che permettesse di partire dall'università per mettere in discussione l'intero assetto sociale»4. Tuttavia, le aspirazioni dell'intera comunità studentesca - riassunte dal Manifesto per un'università negativa, redatto nel giugno 1967 da Renato Curcio e Mauro Rostagno 5 - finirono per estinguersi

nel momento in cui le avanguardie informali del loro movimento uscirono dalla cittadella universitaria, nella quale si erano in gran parte formate, per navigare a vista nella società circostante, con la pretesa di dar consistenza e stabilità a formazioni politiche alternative ai partiti ufficiali della sinistra di classe. Sempre meno interessati alle specifiche rivendicazioni della contestazione studentesca [...], il fondamentale problema che dovettero affrontare nel rapporto con la politica fu tutt'uno con il tentativo di convincere gli operai ad accettare [...] il loro presuntuoso ruolo di forza-guida per la rivoluzione contro il sistema» ${ }^{6}$.

Pur avendo contribuito ai «processi di democratizzazione della società»7, la grande spinta ideale che presiedette all'utopia rivoluzionaria incise soltanto in parte sul tessuto sociale e civile italiano, agevolando comunque quelle trasformazioni che ampliarono le possibilità di accesso al sistema d'istruzione e modificarono in profondità i costumi e la morale. Non c'è dubbio che le ragioni del movimento studentesco siano state perlopiù trascurate

da un sistema politico in grado di tradurre queste spinte in un progetto organico di riforma complessiva delle istituzioni pubbliche. Ma questa opportunità non venne colta dalla classe dirigente e dai partiti che si manifestarono del tutto inadeguati a utilizzare le risorse liberate [...] dal movimento, per ridisegnare i rapporti tra stato e cittadini e ricomporre all'interno di un quadro istituzionale rinnovato le fratture aperte dal ciclo di protesta. Qui sta l'anomalia italiana che ebbe come conseguenze sia l'estenuato prolungamento del conflitto sociale nelle fabbriche e nelle scuole, sia

\footnotetext{
3 VENTRONE, Angelo, op. cit., p. 125.

4 Ibidem.

5 Pubblicato sulla rivista «Lavoro politico», il Manifesto per un'università negativa segnò una tappa fondamentale nel processo di maturazione del movimento studentesco trentino, che si lasciò alle spalle i fermenti antiautoritari delle origini per imboccare la via della «contestazione globale del sistema capitalistico». BALESTRINI, Nanni, MORONI, Primo, op. cit., p. 213.

${ }^{6}$ MARINO, Giuseppe Carlo, op. cit., p. 356.

7 CRAVERI, Piero, op. cit., p. 250.
} 
la trasformazione del collasso del centro-sinistra in una gravissima crisi politica di sistema ${ }^{8}$.

All'alba degli anni Sessanta, Napoli visse una complessa fase di transizione che coincise con la fine dell'esperienza amministrativa di Achille Lauro $^{9}$ - spodestato dalla carica di primo cittadino nell'ottobre del 1962 - e l'ingresso sulla scena politica cittadina del senatore democristiano Silvio Gava, personaggio di primo piano della corrente «dorotea» del partito ed ex ministro del Tesoro nel governo Pella. Il controllo diretto sui sistemi di finanziamento pubblico ${ }^{10}$ e sugli istituti di credito pubblici (il Banco di Napoli) e privati (la Banca popolare di Napoli, la Banca Fabbrocini), gli interessi nel settore edilizio e nei servizi pubblici consentirono alla famiglia Gava di trasformare la sua egemonia economica in strumento di legittimazione del potere politico.

\begin{abstract}
La Cassa per il Mezzogiorno, l'Isveimer costituiranno, per i Gava in particolare [...], uno strumento fondamentale per la gestione politica di un processo di espansione economica e di trasformazione, che resterà caratterizzato dal parassitismo assistito e speculativo, senza mai riuscire a intraprendere la strada dello sviluppo produttivo e innovativo. I Gava rappresenteranno così, in modo esemplare, il volto politico del processo di modernizzazione squilibrata del Mezzogiorno d'Italia, che ha garantito l'espansione assistita dell'economia e la regolazione dei rapporti sociali mediante la
\end{abstract}

\footnotetext{
8 FLORES, Marcello, DE BERNARDI, Alberto, op. cit., p. 250.

9 Il sistema di potere laurino si basava su una «rete complessa, ma piuttosto palese, di legami personali, conoscenze, solidarietà ai livelli più diversi [che] investì in pieno la burocrazia comunale, e diede vita ad un modello di amministrazione agile e consono ai limiti che a Napoli incontrava l'amministrazione della democrazia, contenuta nell'esercizio del voto, poco propensa a quella del controllo e della partecipazione. La vita democratica si surrogava nell'accesso individuale o per gruppi agli interessi, piccoli e grandi, che sorsero intorno alla gestione laurina del Comune. Né, va precisato, la presenza di gravi ritardi e insufficienze (si pensi ad ambiti come l'assistenza sociale, la sanità, la scuola, i trasporti) avrebbe intaccato in quegli anni l'«efficienza» di un sistema che, al contrario, dall'emergenza traeva conferme, alibi, alimento. Le concessioni di suolo pubblico per piccole attività, le assunzioni al Comune, vitali in un contesto di profonda crisi occupazionale, o, per altro verso, la gestione personalistica del fisco comunale, attraverso singole transazioni con i contribuenti e, ancora, la rapidità nell'attribuzione delle licenze edilizie e nella rimozione degli ostacoli formali alle speculazioni, consolidarono i vincoli tra la città ed il partito della Flotta, grazie anche ad una miriade di figure intermedie, di mediatori di questo o quell'esponente "monarchico"». TOTARO, Pierluigi, Il potere di Lauro. Potere $e$ amministrazione a Napoli 1952-1958, Salerno, Laveglia, 1990, p. 19. Per un'analisi incisiva sulla rete di consenso che legittimò la primazia del «Comandante», valga l'esaustivo giudizio contenuto in GALASSO, Giuseppe, Intervista sulla città di Napoli, Roma-Bari, Laterza, 1978, p. 245 .

10 I principali referenti politici di Silvio Gava occupavano posti di rilievo nell'Isveimer, fondazione del Banco di Napoli istituita nel 1938 per l'erogazione di crediti a medio e lungo termine a sostegno delle imprese operanti nelle aree depresse del Mezzogiorno.
} 
direzione e l'uso politico dei flussi di spesa pubblica, indirizzati in misura prevalente verso l'edilizia e le grandi opere infrastrutturali [...] ${ }^{11}$.

La svolta riformatrice che portò nel marzo 1962 alla formazione del primo esecutivo di centro-sinistra lasciò tracce estremamente labili sulla vita amministrativa del capoluogo di regione: le giunte guidate da due esponenti dell'apparato gavianeo, Ferdinando Clemente - in carica per due brevi mandati dal 1963 al 1966 - e Giovanni Principe, sindaco dal 1966 al 1970, non riuscirono a superare i problemi di ingovernabilità che gravavano su Napoli né tantomeno a promuovere il superamento di un latente stallo decisionale. Annota Luigi Mascilli Migliorini:

Tutti i grandi progetti legislativi, dalla [...] legge speciale del '62 alla legge «167» sull'edilizia popolare, alla legge «246» sull'incremento di valore delle aree fabbricabili, che avrebbero dovuto costituire strumenti, sia pur parziali, di intervento programmatore dell'Ente locale nello sviluppo della città, si trasformarono in manifestazioni di inefficienza amministrativa, se non più spesso, addirittura, in occasioni per rafforzare un uso clientelare e privatistico del potere e delle risorse pubbliche. La «strategia delle varianti» al piano regolatore del $1939 \mathrm{si}$ sostituì a un rinnovato e organico piano urbanistico, compiendo l'opera di stravolgimento nella configurazione geologica e territoriale della città inaugurata dalle amministrazioni Lauro. [...] Napoli, in gran misura a causa delle insufficienze della sua classe dirigente, rimase l'unica città a uscire indebolita dallo sviluppo economico italiano degli anni Cinquanta-Sessanta. Solo alcune scelte come gli «stralci», da cui presero il via il Centro direzionale e la Tangenziale, pur lasciando intravedere prospettive speculative, rappresentarono, ovviamente in terreni diversi, indici di una difficoltosa modernizzazione e di una omogeneizzazione con la complessiva realtà del paese ${ }^{12}$.

Le contestazioni all'università Federico II di Napoli, intraprese a metà degli anni Sessanta dalla federazione degli studenti (Orun, Organismo rappresentativo dell'università di Napoli) di concerto con i comitati degli assistenti ${ }^{13}$ e dei docenti ${ }^{14}$, scaturirono dalla denuncia delle pessime condizioni strutturali dell'ateneo. La discussione sul dislocamento delle sedi delle facoltà, condotta dai rappresentanti del

\footnotetext{
${ }^{11}$ BARBAGALLO, Francesco, Napoli fine Novecento. Politici, camorristi, imprenditori, Torino, Einaudi, 1997, pp. 3-4.

${ }^{12}$ MASCILLI MIGLIORINI, Luigi, La vita amministrativa e politica, in GALASSO, Giuseppe (a cura di), Napoli, Roma-Bari, Laterza, 1987, pp. 228-229.

${ }_{13}$ ANAU, Associazione Napoletana Assistenti Universitari.

${ }_{14}$ ANPUI, Associazione Nazionale Professori Universitari Italiani.
} 
corpo accademico e degli universitari in una conferenza dell'aprile 1965, si collegò alla presentazione del progetto, illustrato dal rettore Giuseppe Tesauro all'inizio dell'anno accademico 1960-61, per la costruzione di un nuovo Policlinico nel distretto ospedaliero. Avanzando la richiesta di «una sistemazione unitaria delle sedi universitarie che rendesse possibili i rapporti interdisciplinari» 15 , contenuta nel primo Libro bianco sull'edilizia universitaria a Napoli (aprile 1966), gli studenti partenopei portarono in evidenza le trame speculative che soggiacevano alla costruzione del polo sanitario, per il quale erano stati stanziati 40 miliardi di lire.

La situazione napoletana è tipica della condizione di arretratezza economica del Mezzogiorno. La carenza di un adeguato sviluppo industriale genera da un lato disoccupazione e miseria, dall'altro riversa gli investimenti privati nei settori più immediatamente redditizi come l'edilizia. Dal dopoguerra ad oggi, in particolare, si è sviluppata in maniera eccezionale la speculazione sulle aree edificabili e sui fabbricati. [...] Questi gruppi, legati alla speculazione più sfrenata, hanno trovato, $\mathrm{e}$ trovano tuttora, la loro espressione nelle forze che si sono susseguite al governo della città: dal laurismo al centro-sinistra. [...] Questa massiccia speculazione investe tutti i settori della vita cittadina e in questi ultimi tempi si è resa particolarmente evidente nell'università. L'insufficienza delle vecchie strutture a ricevere il sempre crescente numero di studenti ha dato origine ad uno sviluppo caotico dell'edilizia universitaria: istituti, intere facoltà sono sorte nei vari punti della città dove esigeva cioè l'interesse della speculazione. Quegli stessi gruppi che regolano la vita cittadina hanno determinato la costruzione del Nuovo Policlinico ai Colli Aminei (cioè sui suoli acquistati da Verga, ex preside della facoltà di Medicina) e del Politecnico a Fuorigrotta $[\ldots]^{16}$.

Sullo sfondo delle agitazioni indette nel febbraio 1967 contro la riforma Gui ${ }^{17}$, l'ala radicale dell'Unione goliardica italiana (Ugi) dissentì dalla linea «verticistica»

15 PELLEGRINO, Carmen, '68 napoletano. Lotte studentesche e conflitti sociali tra conservatorismo ed utopie, Tissi, Angelica Editore, 2008, p. 27.

${ }^{16}$ Le mani sulla città. Volantino del Movimento studentesco sulla speculazione edilizia che ha nella costruzione del Nuovo Policlinico il suo punto più clamoroso, Biblioteca Nazionale di Napoli, Archivio del Movimento Studentesco, fondo E. MINICOZZI - R. MUSTO, 50, senza data. 17 Presentata dal ministro Luigi Gui nel maggio del 1965, la riforma dell'ordinamento universitario recepì solo in minima parte le proposte formulate dalla «Commissione d'indagine sullo stato e sullo sviluppo della pubblica istruzione», istituita nell'ottobre del 1962 sotto la presidenza del deputato democristiano Giuseppe Ermini. Il disegno di legge 2314, infatti, ridimensionò le prerogative dei nascenti dipartimenti interdisciplinari, circoscritte al solo coordinamento dei dottorati di ricerca. In seconda battuta, la riforma lasciò completamente in sospeso il nodo dell'autonomia universitaria, riconoscendo al Consiglio universitario nazionale un effimero ruolo consultivo. Il profilo minimalista del "piano Gui», incompatibile con la sempre più stringente esigenza di tutelare il diritto allo studio, agganciandolo all'inserimento 
dell'organizzazione ${ }^{18}$, chiedendo la convocazione dell'assemblea generale. La distanza tra i dirigenti dell'Ugi e la base si ampliò nel mese di marzo: in difformità dalla linea ufficiale della federazione, l'assemblea degli studenti, dei docenti e degli assistenti universitari, convocata per il 17 marzo, licenziò un documento di sostegno alle lotte degli universitari trentini, allontanati con la forza dall'Istituto di Scienze sociali ${ }^{19}$. Peraltro, la direzione assunta dalle mobilitazioni, che sfociarono nella prima occupazione del Palazzo centrale dell'università (26 aprile 1967), non trovò il pieno consenso dei docenti e degli assistenti della facoltà di Medicina:

Gli Assistenti e Professori incaricati della Facoltà di Medicina di Napoli, riuniti in assemblea comune il 28 aprile 1967, constatano come lo sciopero in corso in tutte le Università italiane, indetto dal giorno $21 \mathrm{al} 27$ aprile per protesta contro il progetto di Legge Governativo di riforma universitaria, abbia, nella Sede napoletana, assunto un carattere particolare, deviando dagli scopi e dalle modalità originariamente convenute ${ }^{20}$.

Benché intenzionati a proseguire le agitazioni contro il disegno di legge 2314, il corpo docente mosse alcune obiezioni sulle competenze e sulle funzioni dell'assemblea generale in rapporto alle istituzioni di rappresentanza della facoltà:

2) [...] in alcuni episodi recenti ${ }^{21}$ gli Assistenti ed i Proff. Incaricati di Medicina ravvisano il tentativo di esautoramento dei propri rappresentanti nei direttivi delle

dei giovani laureati nel mercato del lavoro, finì dunque per scatenare il malcontento degli studenti nei principali atenei italiani fin dalla primavera del 1965.

${ }^{18}$ In un documento del settembre 1966, dedicato al tema della rappresentanza, i dirigenti della sezione napoletana dell'Ugi annunciarono il superamento del vecchio assetto istituzionale della federazione, non più intesa come «cartello dei movimenti giovanili»: «Di fronte alle nuove scadenze politiche e ai compiti di rilancio della lotta e del movimento, è necessario all'associazione trasformare la propria struttura organizzativa [...] in movimento sindacale di massa». Cfr. Temi e proposte per la conferenza di organizzazione dell'UGI. Documento per la conferenza dell'Unione Goliardica Italiana, BNN, AMS, f. C. PALERMO, 6, 9/1966, p. 12.

${ }^{19}$ L'intervento delle forze di polizia - autorizzato dal rettore dell'ISST, Mario Volpato - pose fine ad un'intensa settimana di convegni, dibattiti e seminari (12-18 marzo 1967) che la comunità studentesca trentina aveva dedicato alle lotte antimperialiste ed alla denuncia della guerra in Vietnam.

${ }^{20}$ Verbale di assemblea degli assistenti e dei professori incaricati della Facoltà di Medicina di Napoli. Critica dello sciopero del 21-27 aprile, BNN, AMS, f. V. DINI, 6 [mutilo], 28/4/1967, s.n.

${ }^{21}$ Gli estensori del documento si riferiscono all'esito dell'assemblea generale del 26 aprile, in cui fu decisa la prosecuzione ad oltranza delle occupazioni nell'ateneo e della serrata al Policlinico. I docenti e gli assistenti lamentavano l'«opera di denigrazione indiscriminata verso la Facoltà di Medicina, di cui si ignorano patentemente i problemi e [...] una notevole impreparazione ed una impostazione demagogica sia sul piano politico che sul piano tecnico [...]». Cfr. Ibidem. 
associazioni, per la mancata richiesta del loro parere in alcune decisioni interassociative.

3) [Essi] ritengono che il potere decisionale sia stato, nella ultima evoluzione delle agitazioni in corso, totalmente devoluto all'assemblea generale, senza passare per la ratifica preventiva degli Organi direttivi statutariamente eletti ${ }^{22}$.

Lo scollamento tra le rivendicazioni degli attivisti ed il pensiero dei delegati delle commissioni paritetiche istituite nelle facoltà fu acuito dalla dura presa di posizione dell'assise studentesca di Architettura, entrata in sciopero alla fine del mese di aprile:

L’Assemblea Generale degli studenti si costituisce come momento reale della contrattazione del rapporto di forze tra movimento studentesco e centro di potere accademico, che è un rapporto politico. Pertanto, si ritengono sospese tutte quelle commissioni paritetiche che hanno vita fino ad ora nella Facoltà, non essendo stati tali organi capaci di formulare scelte politiche e culturali adeguate [...] al movimento studentesco ${ }^{23}$.

Con la vittoria del nucleo studentesco vicino alle posizioni dei partiti della sinistra ufficiale (PCI, PSI, PSIUP) nel congresso dell'Ugi del maggio 1967, si consumò la scissione con i gruppi di estrazione estremista, che fondarono un mese più tardi l'organizzazione più importante nel contesto delle lotte ingaggiate dagli studenti partenopei nel biennio 1968-69: la Sinistra Universitaria (SU). Rilanciando una «prospettiva rivoluzionaria» e rinnegando nel contempo la «burocrazia dei partiti ufficiali di sinistra», i componenti del collettivo, nelle cui file militarono studenti marxisti-leninisti, filo-trotzkisti e cattolici progressisti, affermarono che «il nostro gruppo deve essere uno strumento efficace di combattimento, in grado di orientare le masse, [...], in particolare le masse studentesche, verso il fronte rivoluzionario con il proletariato [...]»24. Ravvisando la contiguità tra le posizioni dei sindacati e le lineeguida dell’Ugi, Sinistra Universitaria condannò la «sindacalizzazione» del movimento intesa come «"filosofia del pollaio", dove il pollaio è l'università, è universitarismo, è rinuncia ad intervenire nelle contraddizioni di fondo della società, è paura di

22 Ibidem.

23 Assemblea generale degli studenti della Facoltà di Architettura di Napoli, tenutasi il 3 maggio 1967 alle ore 11. Documento di richiesta di trasparenza nella gestione didattica ed amministrativa della Facoltà, BNN, AMS, f. PALERMO, 43, 3/5/1967, p. 1.

${ }^{24}$ La Sinistra Universitaria. Documento della S.U. sui suoi obiettivi e sulla sua organizzazione, BNN, AMS, f. G. BORRELLI, 1, 8/1967, p. 7. 
intervenire dove la lotta diventa lotta di classe» ${ }^{25}$. Per «riuscire a trasformare la sua linea politica in azione di massa»26, l'organizzazione partenopea, sensibilmente influenzata dagli scritti di Lenin, indicò quale primo obiettivo da raggiungere la costruzione di

una struttura [...] chiusa alle masse [...], che deve raccogliere l'avanguardia rivoluzionaria cosciente del movimento universitario napoletano, [basata] sulla concezione bolscevica del partito. [...] Concezione bolscevica significa [...] essere alla testa delle lotte delle masse, non al di sopra delle masse, considerare che noi stessi siamo il prodotto, il risultato dell'azione rivoluzionaria delle masse [...] ${ }^{27}$.

Sulla scia delle proteste in corso nelle università italiane, anche nell'ateneo napoletano i temi dell'internazionalismo e dell'antimperialismo esercitarono una fortissima presa sulla comunità studentesca. La critica al sistema capitalistico elaborata dalla SU si configura come una più approfondita riflessione sul divario tra la prospettiva teorica del processo rivoluzionario (il «programma politico» enunciato dagli studenti) e la sua applicazione pratica (l’istituzione del «partito marxistarivoluzionario»)28.

Questi argomenti avevano suscitato l'interesse del collettivo studentesco della facoltà di Economia e Commercio, che indicò nell'«internazionalismo proletario» il contesto in cui elaborare l'«ipotesi rivoluzionaria»:

Per realizzare questo obiettivo è necessario:

1) stabilire un contatto diretto con la massa studentesca mediante azioni rivendicative [...], così da recuperare gli elementi più coscienti e quelli già disposti al dibattito ed alla discussione di temi di natura politica [...].

2) organizzare gruppi di studio su temi di carattere teorico (i classici del marxismo e del leninismo), finalizzati all'acquisizione di una metodologia di analisi sempre

25 Bollettino della Sinistra Universitaria. Presentazione S.U., BNN, AMS, f. BORRELLI, 6, 7/1967, p. 4.

${ }_{26}$ COLELLA, Francesca, Napoli frontale nel Sessantotto, Napoli, Libreria Dante \& Descartes, 2008, p. 115.

${ }^{27}$ La Sinistra Universitaria, BNN, AMS, f. BORRELLI, 1, 8/1967, p. 4.

${ }_{28}^{28}$ Manca purtroppo una efficace direzione di massa che riesca ad incanalare e generalizzare le varie lotte, dirigendo tutte le energie verso l'abbattimento del capitalismo. Manca un discorso politico sul concreto, che costituisca una solida base per l'azione [...]. Cfr. La Sinistra Universitaria, BNN, AMS, f. BORRELLI, 1, 8/1967, p. 2. 
più corretta ${ }^{29}$ ed inoltre su problemi di politica generale più concreti $[\ldots]$ che permettano la comprensione della situazione politica ed economica italiana ed internazionale $[\ldots]^{30}$.

Questa carica ideale, che peraltro indusse il collettivo studentesco napoletano a contestare la politica sovietica della «coesistenza pacifica» tra socialismo e capitalismo, teorizzata da Nikita Chruščëv nel $1956^{31}$, pervase l'occupazione della sede centrale dell'università, indetta per il 21 ottobre 1967, a pochi giorni di distanza dalla morte di Ernesto «Che» Guevara.

L’inizio della discussione alla Camera sul piano di riforma universitaria (5 dicembre 1967) ${ }^{32}$ coincise con l’inizio della fase più acuta delle contestazioni. L'occupazione delle facoltà di Architettura e di Agraria fu interrotta dall'intervento degli agenti di polizia, su mandato del rettore. La protesta di studenti, docenti incaricati ed assistenti si spostò nella sede centrale dell'ateneo, occupata al termine dell'assemblea generale dell'11 dicembre. Indicando nell'università il centro di «formazione della forza-lavoro», il movimento partenopeo passò in rassegna le anomalie del disegno di legge Gui:

La 2314 risponde ad un processo di riorganizzazione e di ristrutturazione della società capitalistica. Parallelamente ai processi di riorganizzazione aziendale si assiste ad un analogo processo di ristrutturazione dell'organizzazione scolastica. La tripartizione dei titoli (diploma, laurea, dottorato di ricerca) risponde alla domanda di formazione di tre tipi di tecnici immediatamente utilizzabili a livello societario.

A) Diploma e laurea forniscono un adeguato numero di tecnici con una qualificazione a livello minimo e medio, con mansioni esclusivamente esecutive e

\footnotetext{
${ }^{29}$ A tal riguardo, cfr. Lotta antimperialista e coscienza rivoluzionaria. Documento dei gruppi di studio del Movimento Studentesco di Napoli per una radicalizzazione dello scontro politico, BNN, AMS, BORRELLI, 12, s.d.

${ }_{30}$ Volantino del Movimento Studentesco di Economia e Commercio sui seguenti punti: 1) rapporto con le masse 2) studio dei classici del marxismo 3) rapporti con altre organizzazioni studentesche ed operaie, BNN, AMS, f. BORRELLI, 33, s.d.

${ }^{31}$ Presentata nel corso del XX Congresso del PCUS, la dottrina della «coesistenza pacifica» accantonava l'idea di uno scontro militare tra il blocco socialista ed il fronte capitalista, teorizzando nel contempo una competizione pacifica sul piano socio-economico tra Unione Sovietica e Stati Uniti. Sotto le insegne dello slogan «Raggiungere e superare l'America», Chruščëv prospettò il superamento delle economie capitaliste sul fronte della produzione pro capite e la costruzione di una società comunista nell'arco di un ventennio.

${ }^{2}$ Il dibattito parlamentare sulla riforma dell'ordinamento accademico - che, tra l'altro, introduceva il diploma universitario, riconosciuto al termine di un percorso di studi più breve rispetto a quanto previsto per le lauree tradizionali - si arenò letteralmente sulla questione degli obblighi didattici, che peraltro riguardava da vicino molti deputati, schieratisi a difesa del doppio incarico. Cfr. CRAINZ, Guido, Il Paese mancato. Dal miracolo economico agli anni Ottanta, Roma, Donzelli, 2003, p.223, nota 32.
} 
già di fatto subordinate alle esigenze del processo produttivo perché i loro contenuti culturali e di formazione mancano degli strumenti metodologici che diano la possibilità di un intervento critico e quindi consapevole in un intero processo di lavoro;

B) dottorato di ricerca: garantisce la classe dirigente perpetuando i propri quadri, nella dimensione della formazione dipartimentale ${ }^{33}$.

La riflessione si spostò sui limiti dell’insegnamento universitario offerto dall'ateneo napoletano:

Un esempio è fornito dai dati relativi agli studenti immatricolati negli anni accademici 1962-63-64 al corso di laurea in Fisica dell'università di Napoli. Il 40\% ha abbandonato il corso nei primi tre anni dall'iscrizione, un altro $17 \%$ non è riuscito nello stesso periodo a superare alcun esame e solo il $4 \%[$...] ha superato gli esami del primo biennio. [...] Si ha l'impressione, di fronte a questi dati, del fallimento totale dell'università nella sua funzione di istruire34.

Dal consesso generale del corso di laurea in Giurisprudenza partì la richiesta di dimissioni di Tesauro, accusato, dopo l'intervento delle forze dell'ordine nei locali occupati dell’Università centrale (13 dicembre 1967), di non avere «la capacità e la possibilità di colloquiare con le componenti universitarie»:

\begin{abstract}
L'Assemblea ritiene che il professor Giuseppe Tesauro non ha la dignità necessaria a ricoprire l'alto incarico, e pertanto lo invita a rassegnare le dimissioni; si impegna a sostenere la lotta necessaria per costringere il Rettore ad andarsene e chiede che insieme alle sue dimissioni vengano presentate quelle dell'intero Senato accademico35.
\end{abstract}

Seguendo una prassi inedita per la contestazione negli altri atenei della penisola, nel gennaio del 1968 una parte della comunità accademica della Federico II propose

\footnotetext{
33 Mozione approvata dall'assemblea degli studenti, degli assistenti e dei professori incaricati dell'Università di Napoli, BNN, AMS, f. FLORES, 77, 11/12/1967, p. 1.

34 Perché l'Università è in lotta. Fascicolo in cui viene svolta una critica dell'Università $e$ vengono analizzate le caratteristiche del piano Gui, BNN, AMS, f. FLORES, 121, p. 2.

35 Mozione approvata dall'assemblea degli studenti, assistenti e professori incaricati della Facoltà di Giurisprudenza. Documento di opposizione al piano Gui-Codignola; protesta contro il Rettore Tesauro per l'intervento della polizia; richiesta di dimissioni del Rettore e del Senato Accademico; richiesta d'impegno dell'opinione pubblica e dei partiti, BNN, AMS, f. FLORES, $86,15 / 12 / 1967$, s.n.
} 
agli studenti del movimento l'istituzione di un sistema di cogestione attraverso i comitati paritetici. Ogni ipotesi di «collaborazione» con il «baronato» fu seccamente liquidata dalla Sinistra Universitaria alla stregua di una mossa «truffaldina»:

Vecchi campioni dell'autoritarismo - che, negli ultimi decenni, sono stati al servizio di tutti i padroni - hanno scoperto una improvvisa vocazione democratica e lanciano a destra e a sinistra inviti al "dialogo" e al "sereno e fecondo dibattito". Abbiamo visto giornali tradizionalmente al servizio di chiunque comandasse [...] pubblicare sulla loro prima pagina articoli carichi di ogni sorta di promesse purché da parte degli studenti si "collaborasse" con le "autorità accademiche". In questo quadro i vecchi campioni dell'autoritarismo [...] hanno lanciato la proposta dei comitati paritetici in cui professori e studenti (cioè persone dotate di tutti i poteri e persone sprovviste di ogni potere) "decidono" insieme sulla conduzione dell'università ${ }^{6}$.

L'attacco agli insegnanti «rinnovatori» e «democratici», preposti «al soffocamento di ogni centro di contestazione, all'integrazione di ogni possibile opposizione», si accompagna ad un giudizio sferzante sulle «componenti moderate e "governative" degli assistenti e degli studenti, ansiosi [...] di essere ammessi a sedere allo stesso tavolo dei professori e di essere integrati nella struttura del potere accademico»37. L'antagonismo con la sinistra istituzionale si sostanziava pertanto nel pericolo di un riflusso delle agitazioni nei consolidati canali della politica universitaria:

Di fronte a questi vasti movimenti, tutte le forze politiche universitarie tradizionali hanno tentato una contro-offensiva adottando una linea articolata nei seguenti punti:

a) la concorrenza verbale con le forze di sinistra, con parole d'ordine apparentemente avanzate, in modo da riguadagnare la direzione del movimento e ricondurlo alla collaborazione con il potere.

b) Gli appelli dei vari partiti, per ottenere il consenso del movimento all'approvazione di un piano GUI emendato, che avrebbe così perduto le sue caratteristiche reazionarie ed acquistata una improvvisa carica rinnovatrice. Questo tentativo vede spesso alleati la maggioranza governatrice ed il PCI e il PSIUP, come

\footnotetext{
${ }^{36}$ Gli obiettivi della nostra lotta. Volantino della Sinistra Universitaria per l'alleanza con il proletariato, BNN, AMS, f. FLORES, 85, s.d.

37 Bollettino della Sinistra Universitaria, sul bilancio della situazione universitaria e sulle prospettive di lotta, BNN, AMS, f. BORRELLI, 2, gennaio-febbraio 1968, s.n. Cfr. anche $A$ chi serve la collaborazione tra professori e studenti. Volantino del Gruppo di lavoro di Matematica del Movimento Studentesco, BNN, AMS, f. MINICOZZI - MUSTO, 54, s.d.
} 
provato dall'accordo [...] stipulato sugli emendamenti da apportare alla norma sui dipartimenti $[\ldots]]^{38}$.

In parallelo con una nuova campagna di occupazioni, che ripresero all'inizio del mese di febbraio nelle sedi di Agraria, Giurisprudenza, Architettura, Lettere e Filosofia, Ingegneria, i collettivi degli universitari istituirono un ciclo di controcorsi ${ }^{39}$ dedicati allo studio del pensiero leninista ed alla ricostruzione delle principali esperienze di lotta intraprese dai più influenti movimenti italiani ed internazionali. Contestualmente, i portavoce degli universitari iscritti ai corsi di Economia e Commercio e di Lettere e Filosofia deliberarono l'istituzione di un'assemblea plenaria, alla quale sarebbero stati affiancati centri di rappresentanza alternativi ai comitati paritetici, di cui fu proposta la soppressione. Scrivono gli studenti della facoltà di Lettere nella mozione approvata il 18 febbraio 1968:

L'Assemblea di Lettere e Filosofia [...] rileva che nelle lotte di dicembre si è affermata nel movimento universitario la coscienza della sterilità di condurre le proprie lotte su un piano puramente sindacale e corporativo e, pertanto, la necessità di impostare il discorso al livello politico come contestazione globale dell'attuale sistema capitalistico.

Alla luce di queste considerazioni, si ribadisce il rifiuto:

1) alle scelte del comitato di agitazione, all'impostazione della attuale occupazione, in quanto rappresenta il tentativo di una integrazione delle lotte da parte delle forze reazionarie e riformiste;

2) alla proposta dei comitati paritetici, che vengono offerti dalle Autorità accademiche come panacea di tutti i mali dell'università [...].

Propone:

1) centri di controllo dal basso indipendenti dalle centrali politiche ufficiali, e dagli organismi rappresentativi [...];

2) Controcorsi di argomento generale sul problema dei rapporti tra l'Università e la società, formati da gruppi di lavoro organizzati dal basso contro la crisi al vertice dell'ORUN [... $]^{40}$.

\footnotetext{
${ }^{38}$ Lotte studentesche e occupazioni padronali. Volantino della Sinistra Universitaria per denunciare che le agitazioni di dicembre possono essere strumentalizzate dall'ORUN e da altre organizzazioni compromesse con i partiti, BNN, AMS, f. MINICOZZI - MUSTO, 2, febbraio 1968, s.n.

39 Per un approfondimento sugli argomenti trattati nei controcorsi, cfr. i materiali di studio custoditi in BNN, AMS, f. B. ANDRIELLO, 20-29.

40 Mozioni approvate nelle varie Facoltà durante il periodo delle agitazioni. Mozioni della Sinistra Universitaria sulle posizioni unitarie delle assemblee di Economia e Commercio, Lettere e Filosofia, Ingegneria, BNN, AMS, f. BORRELLI, 4, 18/2/1968, s.n.
} 
Tuttavia, la «carenza di direzione nelle agitazioni studentesche»41 denunciata dall'assise di Economia e Commercio fu la spia delle divisioni che ostacolarono l'espansione del movimento studentesco napoletano, sul quale pesarono - secondo il giudizio di Francesco Barbagallo - «i forti connotati ideologici e organizzativi che [tesero] fin dal principio a rinchiudere il movimento di massa dentro rigide strutture»42. Nel marzo del 1968, infatti, il collettivo di Architettura43 si dissociò ufficialmente dalla linea di Sinistra Universitaria, accusata di non aver affatto contribuito alle contestazioni studentesche del mese precedente, avvalorando di converso la «tesi della proletarizzazione degli studenti e dei lavoratori intellettuali» $44 \mathrm{e}$ rimarcando la necessità di abbattere il diaframma che separava le aspirazioni degli studenti in lotta dalla questione operaia. I capisaldi teorici ed ideologici del movimento di Architettura - anticipati dall'istituzione nel marzo 1968 di un controcorso sulla sociologia marxiana, che introdusse nella comunità studentesca della facoltà «una componente religioso-meridionalistica tesa all'educazione-emancipazione delle masse popolari napoletane e meridionali»45 - furono enunciati in un documento redatto dagli occupanti della sede di palazzo Gravina:

I compiti [dell'organizzazione] possono essere raggruppati in tre livelli di discorso, già presenti - sebbene in modo ancora impreciso - anche nelle posizioni assunte in passato dal M[ovimento] S[tudentesco].

Tali livelli riguardano:

a) la facoltà - riorganizzazione dell'attività didattica collettiva, concepita come rifiuto della logica della cogestione, e come ricerca interdisciplinare che, oltre a rappresentare l'apertura di prospettive disciplinari nuove a livello di facoltà, favorisca e faciliti l'estensione del discorso di lotta ad altre facoltà.

b) l'università nel suo complesso - creazione di occasioni stabili di incontro tra rappresentanti delle diverse facoltà, eventualmente allargate a studenti medi - per l'elaborazione, la discussione ed il coordinamento continui delle forme di lotta parziali più adeguati.

\footnotetext{
${ }^{41}$ Ibidem.

42 BARBAGALLO, Francesco, Lotte universitarie e potere accademico a Napoli nella seconda metà degli anni Sessanta, in AGOSTI, Aldo, PASSERINI, Luisa, TRANFAGLIA, Nicola (a cura di), La cultura e i luoghi del '68, Milano, Franco Angeli, 1991, p. 311. 43 MSA, Movimento Studentesco di Architettura, fondato il 23 marzo 1968.

44 COLELLA, Francesca, op. cit, p. 116.

45 BARBAGALLO, Francesco, Lotte universitarie e potere accademico a Napoli nella seconda metà degli anni Sessanta, in AGOSTI, PASSERINI, TRANFAGLIA (a cura di), op. cit., p. 312.
} 
c) la società - intesa come contesto socio-politico, caratterizzato da una determinata struttura di potere, in cui l'università è inserita e del quale essa è uno dei più notevoli e delicati meccanismi ${ }^{46}$.

La fuoriuscita da uno stato di isolamento politico e sociale imponeva al MSA l'acquisizione di

\begin{abstract}
una coscienza di sé non come altro dalle forze sociali che contestano la logica, la direzione, il tipo di sistema in cui l'università è inserita. Ma come forza sociale essa stessa, che, dall'esigenza di un'Università diversa, aperta a tutti, formativa nel senso del più generale e ampio dispiegarsi del valore e della potenzialità dell'individuo, centro di ricerca al reale servizio della collettività, scopre il concreto terreno della lotta alla subordinazione dell’Università [...] alle esigenze del profitto privato 47.
\end{abstract}

Al di là delle dichiarazioni programmatiche e del portato simbolico di alcune proposte (l'abolizione dell'obbligo di frequenza e del voto d'esame, l'istituzione di gruppi di ricerca formati dagli studenti), la federazione di Architettura fu incapace di elaborare un'originale strategia rivendicativa che raccogliesse il consenso della base studentesca. Non a caso, la «riorganizzazione» delle mobilitazioni entrò ben presto in una fase di stallo, ammessa anche dai leader della contestazione nella facoltà:

\begin{abstract}
A Napoli, gli avvenimenti di questi ultimi tempi hanno dimostrato come un discorso troppo politicizzato rischi di non legarsi alle masse degli studenti, soprattutto nelle Facoltà in cui la lotta si è andata sviluppando con maggiore lentezza ed interessa tuttora temi rivendicativi che il Movimento Studentesco, a livello nazionale, ha superato nella sua dinamica contestativa 48 .
\end{abstract}

Tra gli argomenti più dibattuti dalle organizzazioni dei professori incaricati e degli assistenti, spicca l'annosa questione dell'edilizia universitaria, accentuata dal repentino

\footnotetext{
${ }^{46}$ Occupazione di Palazzo Gravina. Documento di Palazzo Gravina sui contenuti validi ed originali che il Movimento Studentesco ha espresso nei mesi passati di lotta, BNN, AMS, f. DINI, 126, 1/4/1968, p. 1. Il documento fu dato alle stampe il 22 novembre 1968.

47 Occupazione di Palazzo Gravina. Documento dell'assemblea di occupazione studentiassistenti della Facoltà di Architettura di Napoli sul bilancio delle lotte del Movimento Studentesco, BNN, AMS, f. DINI, 124, 23/4/1968, s.n.

48 Occupazione di Palazzo Gravina. Volantino dell'Assemblea congiunta studenti e assistenti della Facoltà di Architettura, per avviare un lavoro collettivo sui temi concernenti la qualificazione professionale, la funzione formativa dell'Università $e$ il rapporto tra il Movimento Studentesco e realtà nazionale, BNN, AMS, f. PALERMO, 38, 29/4/1968, p. 2.
} 
incremento delle iscrizioni. In un documento approvato dai delegati dell'ANAU e dell'ANPUI si legge:

\begin{abstract}
L'Assemblea congiunta dell'ANAU e della sezione napoletana dell'ANPUI riunita la sera del 2 aprile $1968[\ldots]$ - denuncia ancora una volta la scarsità dei mezzi finanziari assegnati, anche per l'edilizia, all'Università, frutto di scelte che confermano la immutata sistematica sottovalutazione della funzione dell’Università e delle loro esigenze di ristrutturazione per il progresso civile ed economico-sociale del Paese - rileva [...] l'urgenza di dotare Napoli di un'università adeguata anche nelle strutture edilizie a tali fini di - riafferma pertanto l'esigenza che il problema edilizio dell'Università di Napoli sia risolto in maniera globale [...] e che di conseguenza sia avviata per ora la realizzazione a tempo breve di un'unica nuova sede dell'ateneo [...], strutturata organicamente in maniera adatta alla costituzione ed al funzionamento dei dipartimenti e quindi allo sviluppo dei rapporti interdisciplinari tra Facoltà e dell'unità tra didattica e ricerca49.
\end{abstract}

Interrotta l'occupazione di Palazzo Gravina (17 maggio 1968), la protesta si concentrò nella facoltà di Economia e Commercio, ove gli assistenti entrarono in sciopero (7 giugno 1968) e non parteciparono agli esami della sessione estiva per protestare contro l'assegnazione ad Antonio Gava, figlio del senatore democristiano Silvio, del primo posto nella graduatoria degli assistenti. Nel corso dell'estate, la protesta degli studenti partenopei entrò in una spirale involutiva che del resto caratterizzò anche gli altri gruppi universitari italiani,

a causa di dispute ideologiche che nella loro intransigenza accentuavano divisioni e faziosità tra segmenti diversi del movimento [...]. Si andavano costituendo gruppi più o meno articolati [...], tra i quali emergeva il raggruppamento che attraverso Servire il Popolo avrebbe dato vita all'Unione dei Marxisti-Leninisti, una formazione tra le più intransigenti e dogmatiche $[. . .]^{50}$.

Alla ripresa dell'attività accademica (novembre 1968), tra i docenti incaricati e tra gli assistenti di estrazione radicale si consolidò la volontà di conferire alle proteste un profilo dichiaratamente politico, annunciando lo scioglimento delle due organizzazioni di categoria (ANAU ed ANPUI) e la contestuale istituzione della sezione napoletana

49 Documento dell'Assemblea congiunta ANAU-ANPUI sulle problematiche dell'edilizia universitaria a Napoli e proposte per la soluzione del problema, BNN, AMS, f. DINI, 104, 2/4/1968, pp. 1-2. 50 PELLEGRINO, Carmen, op. cit, p. 111. 
dell'ANDS (Associazione Nazionale Docenti Subalterni). Indicando nel docente unico a tempo pieno il primo obiettivo da conseguire, gli aderenti all'ANDS cercarono altresì di aprire subito il confronto con la classe operaia:

In questo periodo un discorso sull'Università e sulla ricerca gestito dai soli universitari come controparte del governo è un discorso estremamente pericoloso per tutti i rischi corporativi che esso implica, non essendo ancora maturate all'interno dell'Università italiana [...] le condizioni materiali e di coscienza per il verificarsi di una spinta totalmente rinnovatrice da parte della componente non studentesca [...]. Perciò ci siamo mossi non tanto cercando il contatto con gli insegnanti medi ed altre categorie del mondo dell'istruzione pubblica, quanto quello con i sindacati operai, proprio perché riconosciamo di essere legati in qualche modo alle attività produttive, particolarmente nei settori più moderni dell'Universitàs1.

Il collegamento con il mondo del lavoro fu certamente rafforzato dall'attenzione riservata alla questione dei «borsisti»52, figure professionali introdotte nell'ordinamento accademico italiano dalla legge 62/1967. La polemica dell'ANDS riguardava in particolar modo la dequalificazione professionale dei neo-laureati.

L'unica funzione del borsista, soprattutto negli Istituti in cui si svolge lavoro di ricerca professionale, è ormai quella di fornire forza-lavoro al più basso livello di qualificazione per il controllo delle apparecchiature e per l'esecuzione del lavoro di routine. L'uso di tecniche di parcellizzazione del lavoro scientifico, all'interno dei gruppi di ricerca, e la rigida gerarchizzazione delle conoscenze [...] tendono a scaricare sul borsista tutto il lavoro manuale ed esecutivo53.

Nel tentativo di rilanciare la propria azione54, il movimento studentesco napoletano condivise le battaglie degli operai degli stabilimenti della cintura urbana (Italsider, Alfa

\footnotetext{
${ }^{51}$ Per un discorso con i sindacati operai. Documento dell'ANDS sulla necessità dell'incontro con $i$ sindacati operai per inserire i problemi dell'Università nel più ampio processo produttivo e di ristrutturazione della società, BNN, AMS, f. DINI, 65, 1969, p. 2.

${ }^{2}$ Assunti dalle università o dagli istituti di ricerca con contratto biennale, $\mathrm{i}$ «borsisti» erano selezionati tra coloro che avessero conseguito la laurea al massimo da quattro anni accademici. 53 Documento dell'ANDS sulla figura del borsista, BNN, AMS, f. MINICOZZI - MUSTO, 84, [22/12/1968], p. 4 .

54 «I compiti che [...] si pongono come sforzo riorganizzativo dell'intero Movimento Studentesco Napoletano [...] possono grosso modo essere raggruppati in tre livelli di discorso già presenti, anche nelle posizioni assunte in passato dal Movimento e cioè a) la facoltà; b) la scuola nel suo complesso; c) la società». Cfr. Assemblea generale dell'Ateneo napoletano. Documento del Movimento Studentesco Napoletano sull'unità studenti-operai in nome dell'antiautoritarismo, in BNN, AMS, f. DINI, doc. 146, 7/11/1968, pag. 3.
} 
Romeo, Ignis Sud) per il rinnovo dei contratti e sul tema delle «gabbie salariali», partecipando allo sciopero generale indetto per il 7 novembre 1968, e patrocinò le agitazioni nelle scuole superiori. Nucleo di aggregazione delle rivendicazioni dei liceali fu il disegno di legge55 presentato dal nuovo ministro dell'Istruzione, Fiorentino Sullo, che introduceva una capillare rivisitazione dell'esame di maturità. Per la Sinistra studenti medi, questo provvedimento amplificava «la realtà di classe della società borghese che si esplica nella scuola»56. Neppure la disponibilità del ministro democristiano alla rivisitazione della circolare sulle assemblee d'istituto57 (novembre 1968) convinse gli attivisti delle scuole superiori, in quanto le nuove disposizioni «[tendevano] a dare agli studenti l'illusione di un potere che essi in realtà non hanno, in modo che la loro critica tocchi aspetti particolari senza possibilità di una generalizzazione politica [...]»58. In ogni caso, il coordinamento delle proteste nei licei e nelle scuole tecniche partenopee - delegato ai gruppi d'istituto - risultò talvolta discontinuo ed inefficace. Gli attivisti dell'istituto Enrico Fermi, sottolineando «la mancanza di ogni più elementare forma di vita civile associata», si scontrarono con «il qualunquismo più esasperato, che nega ogni rapporto dell'individuo con la

\footnotetext{
55 La riforma dell'esame di Stato, contenuta nel decreto-legge 9 del 15 febbraio 1969, riduceva a due il numero delle prove scritte in programma: il tema d'italiano - comune a tutti i corsi di studio - e il compito specifico per ciascun indirizzo. Il colloquio finale era invece circoscritto a due sole materie, la prima indicata dal candidato, la seconda scelta dalla commissione. A margine di questo provvedimento, fu altresì disposta l'abolizione della sessione autunnale di riparazione. La nuova disciplina degli esami di maturità - che, nelle intenzioni del legislatore, doveva avere carattere «sperimentale» - rimase in vigore fino al 1997. Cfr. «Conversione in legge, con modificazioni, del decreto-legge 15 febbraio 1969, n.9, riguardante il riordinamento degli esami di maturità , di abilitazione e di licenza della scuola media», in Camera dei Deputati - Portale storico, URL:

<http://www.camera.it/_dati/lego5/lavori/stampati/pdf/10460001.pdf> [consultato il 19 luglio 2016].

56 «È evidente che essa [la riforma] non modifica in nessun caso il carattere della scuola italiana come scuola di classe, destinata a garantire l'organizzazione del consenso, la manipolazione delle coscienze, l'accettazione più piena di una società basata sullo sfruttamento e su di una falsa democrazia». Cfr. Denuncia di una strumentalizzazione del Movimento Studentesco. La riforma Sullo. Documento della Sinistra Studenti Medi, in BNN, AMS, f. BORRELLI, 39, 14/4/1969, s.n.

57 Il primo provvedimento sulla convocazione delle assemblee scolastiche fu firmato dal democristiano Giovanni Battista Scaglia, titolare del dicastero dell'Istruzione nel governo Leone (II) dal giugno al dicembre 1968. La circolare concedeva ai presidi la facoltà di autorizzare le riunioni studentesche, a patto che esse si tenessero al di fuori dell'orario curricolare, sotto lo stretto controllo degli insegnanti, e che non degenerassero in atti di intolleranza. La principale modifica apportata da Sullo riguardò l'opportunità di convocare le assise anche negli orari riservati alle lezioni. Cfr. CRAINZ, Guido, op. cit., p. 276.

${ }^{58}$ La Circolare Sullo come tentativo di smobilitazione e di strumentalizzazione del Movimento studentesco della scuola superiore. Volantino della Sinistra Studenti Medi, BNN, AMS, f. MINICOZZI - MUSTO, 29, s.d.
} 
collettività»59. Il gruppo d’istituto del Vittorio Emanuele II annotò la superficialità delle discussioni assembleari, incentrate perlopiù sull'abolizione del voto e degli esami. Più maturo fu invece l'humus politico-ideologico sul quale si articolarono le contestazioni al liceo scientifico Mercalli, intensificatesi nel corso dell'anno scolastico 1969-70:

[...] sin dall'anno scorso, gli studenti del Mercalli uniti a tutte le scuole di Napoli [...] si sono mossi, con la rivendicazione pratica dell'assemblea, sulla parola d'ordine del dibattito politico nella scuola [...]. Sulla linea di questa crescente politicizzazione del movimento le lotte di quest'anno si sono aperte con una viva e spontanea partecipazione che parte dagli studenti del Mercalli a tutta una serie di lotte condotte dai tecnici contro la dequalificazione e quindi contro tutte le manovre ministeriali che sempre avvengono sulla testa degli studenti per piegarci invece alle esigenze di sviluppo dei gruppi industriali, agli interessi cioè della classe dominante ${ }^{60}$.

Le contestazioni che paralizzarono il capoluogo campano dopo l'uccisione di due braccianti ad Avola ( $1^{\circ}$ dicembre 1968) annunciarono la radicalizzazione delle battaglie ingaggiate dalle avanguardie del movimento, entrato a più riprese in conflitto con i militanti di estrema destra del FUAN (Fronte Universitario d'Azione Nazionale), protagonisti fin dal dicembre del 1968 di una serie di gesti sediziosi ${ }^{61}$ ed attacchi ai licei ed alle sedi occupate dell'ateneo ${ }^{62}$. Dopo l'irruzione del 25 gennaio, il «Centro di informazione politica» del movimento partenopeo - che attaccò anche le scelte della Sinistra Universitaria, incapace di prendere «coscienza dei nostri veri interessi» emanò un appello diretto all'intera base studentesca per la formazione di comitati di base autonomi:

59 Chi è il Movimento Studentesco: Documento del M.S. con resoconti dei seguenti gruppi d'istituto: Mercalli, Genovesi, Da Vinci, Della Porta, Vittorio Emanuele II, Umberto, BNN, AMS, f. BORRELLI, 35, s.d., p. 11.

6o Ibidem.

${ }^{61}$ Nella tarda serata del 5 dicembre 1968, una trentina di studenti appartenenti al movimento di estrema destra «Giovane Italia» assaltarono la sede del quotidiano «Il Mattino». Sette di essi furono deferiti all'autorità giudiziaria con le accuse di danneggiamento e lancio di oggetti pericolosi. Cfr. Teppismo a Napoli contro la sede del «Mattino», in «Corriere della Sera», 6 dicembre 1968, p. 2. Cfr. anche Sette denunce a Napoli per l'attacco a «Il Mattino», in «Corriere della Sera», 19 dicembre 1968, p. 2.

62 Un episodio eclatante si consumò durante una riunione degli attivisti del movimento nella sede centrale della Federico II (25 gennaio 1969): un gruppo di militanti del FUAN appiccò un rogo che raggiunse il primo piano dell'edificio ove erano radunati gli studenti. L'assalto all'università fu oggetto di un'interrogazione parlamentare formulata da alcuni esponenti di primo piano del PCI partenopeo (Giorgio Amendola, Massimo Caprara, Giorgio Napolitano). 
Ciò che dobbiamo rifiutare è la violenza, sia diretta che indiretta [...]. Dobbiamo avere la forza per dimostrare che non accettiamo più il controllo che viene svolto su di noi prima dai professori e, quando non è più sufficiente, dall'apparato repressivo dello Stato borghese (polizia, carabinieri, ...). La repressione passa non solo attraverso i professori e la polizia, ma anche attraverso la mistificazione dei contenuti e l'imbrigliamento della nostra lotta da parte dei partiti della sinistra ufficiale [...] Trasformiamoci in attori di quello sviluppo che fino ad oggi ci ha visto vittime. ORGANIZZIAMOCI IN COMITATI DI BASE PER GARANTIRCI AUTONOMIA IN SCELTE E IN DECISIONI POLITICHE63.

Durante l'inverno 1969, la facoltà di Medicina riprese il comando delle mobilitazioni, motivate tanto dall'imminente discussione in consiglio comunale del nuovo piano regolatore ${ }^{64}$ - che, accogliendo le sollecitazioni del rettore, avrebbe completato il processo di smembramento dell'università in tre distinte aree didattiche - quanto dall'impermeabilità delle autorità accademiche alle proposte di rinnovamento presentate dai delegati dell'ANDS: l'insediamento di un consiglio di facoltà aperto anche ai docenti subordinati, la retribuzione degli assistenti volontari e del personale medico delle cliniche interne all'università, la divulgazione delle delibere consiliari. Al termine di uno sciopero protrattosi per ottanta giorni, il 16 aprile 1969 i direttori delle cliniche siglarono gli accordi con gli assistenti ed i professori incaricati, approvandone le richieste più urgenti. Tuttavia, quelle conquiste rimasero soltanto sulla carta: tre mesi più tardi, nel luglio 1969, un nuovo ciclo di agitazioni investì il Policlinico ${ }^{65}$. Anche

63 «Studenti... organizziamoci in comitati di base per garantirci autonomia di scelte e di decisioni politiche». Critica al progetto di legge Sullo, BNN, AMS, f. U. LEPORE, 36, gennaio 1969, p. 1.

64 Adottato nel marzo del 1970 dall'assise cittadina, il nuovo Piano regolatore della città fu profondamente rivisto dal Ministero dei Lavori pubblici - che ne dispose l'attuazione con D.M. 1829 del 31 marzo 1972 - con l'obiettivo di non stravolgere l'assetto urbanistico della città. Annota Enrico Cardillo: «Oltre a diversi interventi di trasformazione ritenuti strategici per una Napoli metropoli dei servizi, il piano affronta tre fondamentali questioni: la delocalizzazione dell'aeroporto a nord nonché quella dei grandi complessi industriali a est e ad ovest della città [...]. Delle aree petrolchimiche ad oriente e dell'Italsider ad occidente (da ampliare, secondo il piano adottato nel 1970) il 30\% viene destinato ad attrezzature pubbliche a seguito delle modifiche imposte dal Consiglio Superiore dei Lavori Pubblici, mentre il restante 70\% viene sottoposto a riqualificazione e destinato ad attività produttive non inquinanti e ad alto numero di addetti». CARDILLO, Enrico, Napoli, l'occasione post-industriale. Da Nitti al piano strategico, Napoli, Guida, 2006, pp. 40-41.

65 Scrivono i delegati dell'ANDS in un documento che annunciò la ripresa della serrata: «4 MILIARDI DI LIRE è il fatturato del Policlinico di Napoli, realizzato da pochi CATTEDRATICI con l'uso di pubbliche risorse e da essi stessi gestito SENZA CONTROLLI. A tal fine ESSI usano anche il lavoro di 500 ASSISTENTI VOLONTARI E MEDICI INTERNI NON RETRIBUITI. $\underline{\text { A }}$ nulla è servito il "memorabile patto" del 16 aprile [...]. Oggi improvvisamente si scopre che mancano i fondi per la retribuzione pattuita. Al contrario i fondi non sono mai mancati per la disgregazione dell'Università [...] e non mancano per portare avanti i lavori al nuovo Policlinico, 
in questo caso, però, tutte le proposte dei medici subalterni (democrazia interna, priorità dell'attività scientifica e didattica sull'assistenza sanitaria, accentramento delle strutture universitarie) furono completamente rigettate: con una decisione senza precedenti nella storia dell'università italiana, nel 1971 il Senato accademico della Federico II dispose lo sdoppiamento della facoltà di Medicina. La collaborazione tra l'ANDS ed il movimento studentesco napoletano raggiunse il suo apogeo nel corso della primavera 1969. Nel solco delle battaglie contro la riforma Sullo ed il nuovo PRG (Piano Regolatore Generale), dalle quali scaturì la pubblicazione del Secondo libro bianco sull'edilizia universitaria a Napoli, la definizione di una strategia comune fu sanzionata dalla clamorosa protesta dei docenti subalterni del corso di laurea in Ingegneria, che disertarono la riunione del consiglio di facoltà convocata per il 24 maggio 1969. La mozione approvata dall'assemblea generale è un atto d'accusa contro «il potere assoluto dei cattedratici all'interno delle Facoltà», tema già assurto a baluardo della contestazione studentesca.

Non è infatti pensabile [...] che [...] l'azione del docente subalterno possa svolgersi in piena libertà e autonomia, quando le decisioni da adottare toccano interessi di persone da cui egli dipende e materialmente e per la produzione scientifica, strettamente legata alla sua carriera [...]. Si giunge così alla massima dequalificazione delle decisioni prese dal Consiglio di Facoltà, le quali scaturiscono non da un'analisi, sia pure superficiale, dei problemi della Facoltà, ma da interessi personali di gruppi di professori [...] oppure da rigurgiti demagogici volti alla cattura del consenso delle masse studentesche ${ }^{66}$.

Nell'assise del 12 giugno, l'obiettivo dei professori subalterni si spostò sulla gestione del ciclo biennale di studi propedeutici:
Al tempo in cui ancora si riteneva possibile una collaborazione con il C[onsiglio] d[i] F[acoltà], gli studenti e i docenti sollecitarono più volte un'analisi delle possibili soluzioni alla crisi sempre più drammatica del biennio, soprattutto allorché la Facoltà venne a disporre del finanziamento di Lire 2.180.000.000.

destinato da TESAURO-BEGUINOT e C. a triplicare l'attuale fatturato con addirittura 3000 posti-letto [...]». Cfr. La lotta universitaria al Policlinico. L'ANDS sez. di Napoli si batte per un'Università nuova fondata sul diritto allo studio e sull'unità didattica-ricerca, BNN, AMS, f. LEPORE, 351, 10/7/1969, s.n.

66 Volantino dell'Assemblea dell'ANDS sezione di Napoli, Assemblea della Facoltà di Ingegneria per denunciare la mancanza di autonomia e di peso decisionale dei docenti $e$ ricercatori subalterni, BNN, AMS, f. DINI, 48, 23/5/1969, s.n. 
Tale responsabile iniziativa fu disinvoltamente aggirata per dar luogo a provvedimenti che rispondevano ad interessi settoriali e che vennero motivati con una loro assoluta indilazionabilità [...]. Per questi giochi personalistici si sono calpestati i diritti di alcune migliaia di studenti del biennio ${ }^{67}$.

A ben vedere, però, dal rapporto con i docenti dell'ANDS non sortì il rilancio della linea politica del movimento studentesco napoletano, peraltro già lacerato al suo interno dai conflitti tra simpatizzanti di Potere Operaio ed i sostenitori dell'Unione italiana marxisti-leninisti: ridimensionando la portata delle rivendicazioni originarie, le avanguardie della Sinistra Universitaria predilessero tendenze settarie (le battaglie contro l'imperialismo americano e contro l'uso classista della scienza nelle società capitalistiche, l'approfondimento del pensiero leninista) al confronto con il mondo operaio nella stagione dei rinnovi contrattuali (autunno 1969) ed alla discussione dei problemi - in larga parte irrisolti - della comunità studentesca del capoluogo campano. Pare altresì evidente che il declino del movimento napoletano sia ascrivibile al carattere pressoché disomogeneo delle proteste indette dalle singole facoltà ed all'assenza di un autentico legame con i problemi della città, rimasti sovente sullo sfondo delle contestazioni. All'alba degli anni Settanta, osserva Francesco Barbagallo,

l'università rimane, anche se in forma ridotta, un luogo di iniziativa politica; ma l'attenzione tende qui a spostarsi sui problemi interni di riorganizzazione, in connessione con i parziali progetti governativi di modifica del contesto universitario. Si riduce progressivamente l'influenza degli studenti, mentre le tendenze più definitivamente riformistiche di docenti e ricercatori si concentrano all'interno dell'università e trovano uno sbocco parziale nella Cgil-scuola, attiva dal 1970 nelle università napoletane ${ }^{68}$.

Un altro, interessante risvolto delle lotte studentesche fu senz'altro l'impegno dei cattolici, nel triennio 1966-1969, a sostegno della popolazione disagiata del rione Don Guanella, nella periferia settentrionale del capoluogo campano. L'intervento del volontariato cattolico al servizio delle famiglie degli «ex-baraccati», alloggiate nelle

\footnotetext{
${ }^{67}$ Allegato B alla Mozione approvata all'unanimità nell'Assemblea del 17 dicembre e letta alla riunione del Consiglio di Facoltà del 18 dicembre 1969. Documento dell'ANDS - Facoltà di Ingegneria per richiedere un più ampio dibattito pubblico sulla didattica, BNN, AMS, f. DINI, 62, [12/6/1969], p. 1.

68 BARBAGALLO, Lotte universitarie e potere accademico a Napoli, in AGOSTI, Aldo, PASSERINI, Luisa, TRANFAGLIA, Nicola (a cura di), op. cit., pp. 319-320.
} 
palazzine dell'Istituto Autonomo Case Popolari (IACP), si configurò in prima battuta come una denuncia dello stato di degrado in cui versava l'intera frazione:

Il quartiere era ed è tuttora da completare e si presenta carente anche in aspetti di prima necessità. Così per esempio le strade sono da terminare [...], la illuminazione e la pulizia insufficienti. Non esiste nessuna farmacia, la più vicina si trova ad una distanza di 20 minuti di cammino. Non c'è nessun ambulatorio e posto di pronto soccorso $^{69}$.

Documentando la loro attività, articolata in una serie di corsi di cultura popolare e di educazione civica, i giovani attivisti certificarono la difficoltà di superare il clima di diffusa ostilità che accompagnò la loro missione educativa:

\begin{abstract}
Noi pensiamo che $\mathrm{i}$ dati negativi di questa esperienza ${ }^{70}$ confermano l'impreparazione dell'ambiente a questo tipo di lavoro e la mancanza di interesse verso tutto ciò che possa essere una apertura ad altri argomenti che non sembrano risolvere immediatamente e concretamente i loro problemi più urgenti $[\ldots]^{71}$.
\end{abstract}

Eppure, nel documento licenziato alla fine del secondo ciclo di lavoro nel rione Guanella, terminato nel luglio del 1969, i rappresentanti del nucleo di volontariato registrarono le pur parziali aperture della popolazione a questi progetti: «Guardando questi tre anni di attività, ci sembra che, anche se molto lentamente, qualcosa sta cambiando. Alcune persone sentono ormai come cosa propria il quartiere, avvertono di far parte di una comunità in via di sviluppo $[\ldots]{ }^{\prime 7} 7^{2}$.

Alle spinte movimentiste che connotarono la prima fase delle mobilitazioni imperniate tanto sulla denuncia delle manovre speculative sul territorio, quanto sulla difesa del diritto allo studio - non seguì tuttavia la costruzione di una piattaforma politica condivisa che coinvolgesse attivamente la città, rimasta perlopiù spettatrice delle contestazioni. Inoltre, la tardiva scelta di sganciarsi dalle ridondanti parole d'ordine dell'epoca (antiautoritarismo, internazionalismo, antimperalismo) e di avvicinarsi alle ragioni della comunità operaia non giovò di certo alle centrali studentesche partenopee, del tutto incapaci di allargare la propria base di consenso al

${ }^{69}$ Relazione del lavoro svolto nel rione D. Guanella. Documento del Volontariato cattolico, BNN, AMS, f. A. L. FARRO, 3, 26/7/1968, p. 2.

${ }^{70}$ Annota il gruppo del volontariato cattolico: «La frequenza non ha superato le 6-7 persone nei casi di maggiore partecipazione». Ibidem, p. 7.

${ }^{71}$ Ibidem.

${ }^{72}$ Relazione del lavoro svolto nel rione D. Guanella (settembre 1968-luglio 1969). Documento del Volontariato cattolico, BNN, AMS, f. FARRO, 6, p. 4. 
di fuori del perimetro accademico. In ogni caso, sarebbe superficiale presentare il Sessantotto napoletano come un'imitazione malriuscita delle proteste che scossero i maggiori atenei italiani: al contrario, questo lavoro ha cercato di mettere in risalto la vivacità di un ambiente universitario considerato a torto marginale sulla scena delle contestazioni. 


\section{* L'autore}

Carmine Marino (Battipaglia, Salerno, 1986) ha conseguito la laurea in Storia e Civiltà Europee presso l'Università della Basilicata con una tesi in Storia contemporanea dal titolo Per una storia del Sessantotto. Abilitato all'insegnamento di materie letterarie negli istituti di istruzione secondaria di I e II grado, ha partecipato nel settembre 2015 alla XVIII Settimana di Studi tardoantichi e romanobarbarici, organizzata a Monte Sant'Angelo (Foggia) dall'Università di Bari.

URL: < http://www.studistorici.com/progett/autori/\#Marino >

\section{Per citare questo articolo:}

MARINO, Carmine, «Documenti inediti per la cronaca e per la lettura storica del Sessantotto napoletano», Diacronie. Studi di Storia Contemporanea : Stato, costituzione e democrazia, 29/09/2016, URL:< http://www.studistorici.com/2016/09/29/c-marino_numero_27/ >

Diacronie Studi di Storia Contemporanea $\widehat{\beta}$ www.diacronie.it

Risorsa digitale indipendente a carattere storiografico. Uscita trimestrale. redazione.diacronie@hotmail.it

Comitato di redazione: Jacopo Bassi - Luca Bufarale - Elisa Grandi - Antonio César Moreno Cantano - Deborah Paci - Fausto Pietrancosta - Alessandro Salvador - Matteo Tomasoni - Luca Zuccolo

Diritti: gli articoli di Diacronie. Studi di Storia Contemporanea sono pubblicati sotto licenza Creative Commons 3.0. Possono essere riprodotti e modificati a patto di indicare eventuali modifiche dei contenuti, di riconoscere la paternità dell'opera e di condividerla allo stesso modo. La citazione di estratti è comunque sempre autorizzata, nei limiti previsti dalla legge. 